\title{
Influence of a Direct-Conversion Receiver Model on the Performance of Detectors for Spectrum Sensing
}

\author{
Dayan Adionel Guimarães, Elivander Judas Tadeu Pereira
}

\begin{abstract}
An implementation-oriented receiver model for centralized data-fusion cooperative spectrum sensing was proposed a few years ago to assess the performances of the energy detector and some eigenvalue-based detectors. The model is grounded on a direct-conversion receiver whose main influences on the sensing performance have been found to be the direct-currentoffset and the automatic gain control. In this paper we improve the referred model and use it to assess the performances of state-of-the-art blind detectors whose computations of the test statistics are among the least complex known to date. These detectors are the Gerschgorin radii and centers ratio (GRCR), the Gini index detector (GID), the Pietra-Ricci index detector (PRIDe), and the locally most powerful invariant test (LMPIT). The energy detector (ED) is also included as a benchmark. It is shown that the performances of all detectors are overestimated if the conventional model (in the sense of signal processing operations not oriented by receiver implementation aspects) is adopted. The ED is the detector whose performance is the most affected by the operations made in the implementation-oriented model. The other detectors are affected in quite similar ways, with an advantage of the PRIDe in most of the situations analyzed.
\end{abstract}

Keywords-Cognitive radio, DC-offset, direct-conversion receiver, spectrum sensing.

\section{INTRODUCTION}

$\mathbf{T}$ $\mathrm{HE}$ demand for new telecommunications services has been the main research driver of new technologies, as can be seen, for example, in the recent advances involving the Internet of things (IoT) and the fifth generation (5G) of communication networks, as well as the discussions already started on the sixth generation (6G). However, it is necessary to overcome the obstacle of radio-frequency (RF) spectrum scarcity to allow the deployment of new wireless communications services, as a large amount of RF bands would be necessary to accommodate the high number of transmitters and receivers foreseen for IoT, $5 \mathrm{G}$ and $6 \mathrm{G}$ networks. Such scarcity is due to the fact that, in the current fixed bandwidth allocation policy, the usage right is given only to the contracting user, also called licensed, incumbent or primary user (PU).

It is believed that the fixed spectrum allocation policy will not be adequate for the expansion of wireless communications systems and services. A new and more appropriate policy for dynamic spectrum sharing (DSS) or dynamic spectrum access (DSA) is needed, aiming at exploiting the fact that the RF

Dayan A. Guimarães and Elivander J. T. Pereira are with the National Institute of Telecommunications (Instituto Nacional de Telecomunicações, Inatel), Santa Rita do Sapucaí, MG, Brazil. Phone:+55(35)34719227, e-mail: dayan@inatel.br, elivander@mtel.inatel.br.

This work was partially supported by RNP, with resources from MCTIC, Grant No. No 01245.010604/2020-14, under the 6G Mobile Communications Systems project of the Radiocommunication Reference Center (Centro de Referência em Radiocomunicações, CRR) of Inatel. DOI: 10.14209/jcis.2021.19. spectrum is actually underutilized, given that much of the time and in certain regions there are allocated frequency bands that are unoccupied [1], [2].

In the dynamic spectrum allocation policy, it is accepted that an unlicensed user, also called secondary user (SU), can use a frequency band already licensed to the primary network. The SU transmissions can be carried out both simultaneously with PU transmissions, as long as no harmful interference is caused to the primary network, or in a non-overlapping manner, taking advantage of transmission opportunities in licensed bands that are momentarily unoccupied.

The concept of cognitive radio (CR) arose in such context [3]. A CR is an intelligent transceiver device that possesses several sophisticated attributes related to cognition, allowing it to adapt to the environment and to the network in which it is inserted. Among the CR attributes, we highlight the spectrum sensing [4], [5], through which an SU transceiver device can identify momentarily vacant frequency bands, allowing a shared spectrum use between the primary and the secondary networks. Thus, spectrum sensing can be considered one of the main enablers of DSA.

The CR technology already takes place in current networks, and is also envisaged as part of future ones. For example, since the Release 13 of the $3^{\text {rd }}$ Generation Partnership Project (3GPP) recommendations, the unlicensed operation for mobile communications implements the listen before talk (LBT) protocol, which is in fact a realization of spectrum sensing to protect Wireless Fidelity (WiFi) communications from Long Term Evolution (LTE) interference [6]. Recent works on 5G and beyond are also in ongoing development, considering cognitive physical layers for operation in licensed bands by means of DSA [7].

\section{A. Reviewing spectrum sensing concepts}

The spectrum sensing process is a binary hypothesis test in which the decision upon the presence (hypothesis $\mathcal{H}_{1}$ ) or absence (hypothesis $\mathcal{H}_{0}$ ) of the PU signal in the sensed band is made by comparing a test statistic (also called decision variable) with a decision threshold whose value is set according with the desired spectrum sensing performance. Wellknown test statistics are based on energy detection, matched filtering, cyclostationary feature detection, and eigenvaluebased detection [4], [5].

In energy detection, which is the simplest spectrum sensing technique known so far, the test statistic is a measure of the energy of the signal received during the sensing interval, which differs from the absence to the presence of the PU 
signal. Matched filtering applies a receive filter matched to the PU signal waveform, which renders it a high implementation complexity, but optimal performance in some cases. The high complexity is also a characteristic of the cyclostationary feature detection, which explores the cyclostationarity of the PU signal to distinguish it from noise, which is not a cyclostationary random process. Eigenvalue-based detection makes use of the eigenvalues of the received signal covariance matrix to detect the presence of the PU signal, being also complex mainly due to the need of computing the covariance matrix and their eigenvalues.

When spectrum sensing is made by each SU independently of the other SUs, unreliable decisions may result, mainly due to multipath fading, shadowing and the hidden terminal problem. Cooperative spectrum sensing (CSS) comes as a more reliable solution that explores the spatial diversity gain achieved by SUs in different locations [4], [5]. The CSS is made by a group of SUs that collaborate, jointly seeking for vacant RF bands.

The CSS can be centralized or distributed. The centralized CSS processes the samples collected by the SUs or the local decisions made by them, which are forwarded to a fusion center (FC) where the final global decision on the sensed band occupancy state is made. When collected samples are sent to the FC, it is referred to as a data-fusion CSS. When the local decisions are sent to the FC, a decision-fusion CSS takes place. In distributed CSS, the SUs in cooperation share their decisions and the global decision is jointly made under some form of consensus. In both approaches, when a global decision is made in favor of a vacant band, it is broadcast to the SUs to allow the subsequent DSA. An ordinary access protocol can be adopted in the DSA phase.

The spectrum sensing performance is often measured by means of the probability of false alarm, $P_{\text {fa }}=\operatorname{Pr}\left\{T>\gamma \mid \mathcal{H}_{0}\right\}$, and the probability of detection, $P_{\mathrm{d}}=\operatorname{Pr}\left\{T>\gamma \mid \mathcal{H}_{1}\right\}$, where $\operatorname{Pr}\{\cdot\}$ denotes the probability of occurrence of the underlying event, $T$ is the test statistic formed according with the adopted detection technique, and $\gamma$ is the decision threshold.

It is desirable to have a low $P_{\text {fa }}$ to increase the chance of opportunistic spectrum access by the SUs, which increases the secondary network data throughput. A high $P_{\mathrm{d}}$ is also targeted so that the primary network is protected from interference caused by the secondary network when it mistakenly detects a vacant band and uses it.

Unfortunately, the objectives of increasing $P_{\mathrm{d}}$ and reducing $P_{\text {fa }}$ are concurrent, which means that increasing the first (lowering the decision threshold) also causes the second to increase; and the reduction of the second (increasing the decision threshold) also causes the reduction of the first. In practice, a trade-off solution ruled by a standard is typically adopted. For example, the IEEE 802.22 standard establishes $P_{\text {fa }} \leq 0.1$ and $P_{\mathrm{d}} \geq 0.9$ in a worst case scenario regarding the detection of digital television (DTV) signals [8].

\section{B. Related research and contributions}

In [9], a realistic receiver model for centralized data-fusion CSS has been proposed. The model is grounded on a direct- conversion architecture, claimed to be a more adequate alternative to be applied when assessing the performance of spectrum sensing techniques. The motivation behind the proposal of such model was the fact that there were (and still there is) a lack of research initiatives that consider the influence of typical receiver circuitry, adapted as a spectrum sensor, on the performance of the sensing process. All research publications verified by the authors consider that the samples collected by an SU device are free from any circuit-related impairment when they are processed by the SUs to yield local decisions (in the case of a decision-fusion CSS) or when they are processed in the FC (if a data-fusion CSS takes place).

It has been concluded in [9] that the spectrum sensing performance under the conventional model might be overestimated when compared with the so-called implementationoriented model. The main process that degrades the sensing performance is the automatic gain control. The direct-currentoffset (DC-offset), the quantization noise and filtering also influence performance, but in a smaller amount when typical residual DC-offset (after DC-offset compensation) is considered, when the number of quantization bits is above 3 , and when the filter bandwidth is not too small, i.e. when the sensed channel is not too narrow.

The detection techniques analyzed in [9] were the energy detector (ED), and the eigenvalue-based detectors: generalized likelihood ratio test (GLRT), maximum-minimum eigenvalue detection (MMED), also known as eigenvalue ratio detection (ERD), and maximum eigenvalue detection (MED), also known as Roy's largest root test (RLRT).

Recently, some low-complexity detection techniques have been proposed. Among them, we highlight the locally most powerful invariant test (LMPIT) [10], the Gerschgorin radii and centers ratio (GRCR) detector [11], the Gini index detector (GID) [12], and the Pietra-Ricci index detector (PRIDe) [13]. Due to the fact that the test statistics of these detectors are formed via simple operations on the elements of the received signal sample covariance matrix (SCM), the computational cost to calculate them are slightly higher than in the case of the ED. However, the need for estimating the noise variance to establish the decision threshold in the ED eventually turns its implementation complexity even higher than in the case of the LMPIT, the GRCR, the GID, and the PRIDe.

Besides having low implementation complexity, the detectors LMPIT, GRCR, GID and PRIDe are robust against received signal and noise power variations over time, attain the constant false alarm rate (CFAR) property, are capable of outperforming many detectors in a variety of circumstances of practical interest, and are blind in the sense that neither any PU signal characteristics nor the noise variance information are needed to form the test statistics. We stress the fact that the ED is semi-blind, since it makes use of the noise variance information, although it does not demand the knowledge of the PU signal characteristics.

In this paper we improve the implementation-oriented receiver model proposed in [9], also improving the sensing channel model with respect to the one adopted in [9]. We then apply these models to assess the performances of the detectors LMPIT, GRCR, GID, PRIDe and ED. The main 
receiver-related model improvements refer to modifications in the order of signal processing operations aiming at better matching actual receiver structures, also removing normalization operations made on the noise and signal powers and on the channel gain, which in [9] led to undesired changes in the corresponding long-term statistics. The sensing channel model improvement accounts for a morphology-dependent random Rice factor, and time-variant noise and received signal levels.

It is worth emphasizing that this article does not explore any circuit-level real implementation of the spectrum sensing. Instead, it proposes a model for assessing the spectrum sensing performance via computer simulations. The model is based on a receiver circuit architecture that is considered the most commonly adopted choice in modern transceiver designs, such as software-defined radios (SDRs).

The remainder of the paper is organized as follows: Section II presents the conventional signal model, taking into account the improvements in the sensing channel model. The original implementation-oriented model is briefly described in Section III. Section IV is devoted to the improved receiver model. The test statistics associated with the detectors LMPIT, GRCR, GID, PRIDe and ED are addressed in Section V. Section VI presents the computer simulation results, and Section VII concludes the work.

\section{Conventional SignAL MODEL}

The centralized CSS with data-fusion is accomplished by $m$ cognitive SUs, each one collecting $n$ samples of the signal received from $s$ PU transmitters during each sensing interval. At the FC, these samples form the matrix $\mathbf{Y} \in \mathbb{C}^{m \times n}$, which is given by

$$
\mathbf{Y}=\mathbf{H X}+\mathbf{V} .
$$

Under the hypothesis $\mathcal{H}_{0}$ the primary signal is absent in the band of interest, that is $\mathbf{Y}=\mathbf{V}$. Under the hypothesis $\mathcal{H}_{1}$ the primary signal is present, i.e, $\mathbf{Y}=\mathbf{H X}+\mathbf{V}$. In this equation, the $n$ samples related with the signals transmitted by the $s$ PUs are arranged in the matrix $\mathbf{X} \in \mathbb{C}^{s \times n}$. These samples are zero-mean complex Gaussian random variables whose variance is determined according to the average signal-to-noise ratio (SNR) across the SUs. The Gaussian distribution for the primary signals is adopted due to the fact it appropriately describes the envelope fluctuations of typical modulated and filtered digitally-modulated signals [14].

The channel matrix $\mathbf{H} \in \mathbb{C}^{m \times s}$ in (1) has elements $h_{i j}$, for $i=1,2, \ldots, m$ and $j=1,2, \ldots, s$, representing the flat channel gains between the $j$-th PU and the $i$-th SU. These gains are constant during the sensing interval and independent and identically distributed (i.i.d.) between consecutive sensing rounds. The flat fading assumption means that the PU signal bandwidth is smaller than the coherence bandwidth of the sensing channel. The constant gains model a slow fading, meaning that the sensing interval is smaller than the coherence time of the channel. The independence of successive fading realizations means that the interval between consecutive sensing rounds is larger the the coherence time of the sensing channel. This channel matrix is given by

$$
\mathbf{H}=\mathbf{G A},
$$

where $\mathbf{A} \in \mathbb{C}^{m \times s}$ is formed by complex-valued Gaussian random variables $a_{i j}$ with mean $\sqrt{\kappa_{i j} /\left(2 \kappa_{i j}+2\right)}$ and variance $1 /\left(\kappa_{i j}+1\right)$, yielding $\mathbb{E}\left\{a_{i j}^{2}\right\}=1$, where $\kappa_{i j}$ is the Rice factor $^{1}$ of the channel between the $j$-th PU and the $i$-th SU. To model extremes of realistic scenarios, $\kappa_{i j}=10^{K_{i j} / 10}$, where $K_{i j}$, in $\mathrm{dB}$, is Gaussian distributed with mean $\mu_{K}$ and standard deviation $\sigma_{K}$. For urban areas, $\mu_{K}=1.88 \mathrm{~dB}$ and $\sigma_{K}=4.13 \mathrm{~dB}$. For rural and open areas, it follows that $\mu_{K}=2.63 \mathrm{~dB}$ and $\sigma_{K}=3.82 \mathrm{~dB}$ [15].

Unequal (nonuniform) received signal power levels across the SUs can be considered. This is modeled by setting the gain matrix $\mathbf{G} \in \mathbb{R}^{m \times m}$ as

$$
\mathbf{G}=\operatorname{diag}\left(\sqrt{\frac{\mathbf{p}}{p_{\mathrm{avg}}}}\right),
$$

where the vector $\mathbf{p}=\left[p_{1}, p_{2}, \ldots, p_{m}\right]$ contains the received signal powers across the SUs, and $p_{\text {avg }}=\frac{1}{m} \sum_{i=1}^{m} p_{i}$ is the average signal power over all SUs. Since the average channel power gain is unitary, without loss of generality, each PU transmits with a constant power given by $p_{\text {avg }} / s$.

If unequal and time-varying received signal powers are assumed, then $p_{i}$ is uniformly distributed over the interval $\left[\left(1-\rho_{\mathrm{S}}\right) p_{\mathrm{avg}},\left(1+\rho_{\mathrm{S}}\right) p_{\mathrm{avg}}\right]$ in each sensing round, where $0 \leq \rho_{\mathrm{S}}<1$ is the configurable fractional signal power variation about the average.

In the case of unequal and time-varying noise levels, the elements in the $i$-th row of the matrix $\mathbf{V} \in \mathbb{C}^{m \times n}$ in (1) are i.i.d. Gaussian noise samples with zero mean and variance $\sigma_{i}^{2}$, which is itself a random variable uniformly distributed over the interval $\left[\left(1-\rho_{\mathrm{N}} / 2\right) \sigma_{\mathrm{avg}}^{2},\left(1+\rho_{\mathrm{N}}\right) \sigma_{\mathrm{avg}}^{2}\right]$ in each sensing round, where $0 \leq \rho_{\mathrm{N}}<1$ is the fractional noise power variation about the average, and $\sigma_{\mathrm{avg}}^{2}=\frac{1}{m} \sum_{i=1}^{m} \sigma_{i}^{2}$ is the average noise power across the SUs.

Thus, the received SNR, in $\mathrm{dB}$, averaged over all SUs, is

$$
\mathrm{SNR}=10 \log _{10}\left(\frac{p_{\mathrm{avg}}}{\sigma_{\mathrm{avg}}^{2}}\right) .
$$

Given $\mathbf{Y}$ at the FC, the SCM of order $m$ is computed as

$$
\mathbf{R}=\frac{1}{n} \mathbf{Y} \mathbf{Y}^{\dagger},
$$

where $\dagger$ denotes complex conjugate and transpose.

In [9], the channel matrix represents a Rayleigh fading channel. Here, it represents a more practical-appealing sensing channel producing Ricean fading with random Rice factor whose mean and standard deviation are environmentdependent. Moreover, the received signal and noise powers are fixed in [9], whereas they may be varied in the present model to account for different distances between the SUs and the PUs and different thermal noise levels across the SUs receivers due to different temperatures and inherent circuit uncalibration.

\footnotetext{
${ }^{1}$ In a multipath fading channel, the Rice factor is the ratio between the power in the dominant (specular or line-of-sight) multipath component and the power in the remaining ones. If $\kappa_{i j}=0$, the Ricean fading specializes to the Rayleigh fading. If $\kappa_{i j} \rightarrow \infty$, a pure additive white Gaussian noise (AWGN) channel results. For practical purposes, an almost-pure AWGN channel is observed if $\kappa_{i j}>10$.
} 


\section{THE ORIGINAL IMPLEMENTATION-ORIENTED MODEL}

The receiver architecture whose the model proposed in [9] is based is shown in Fig. 1. It suits to each SU receiver in the decision-fusion $\mathrm{CSS}$, or to the $\mathrm{FC}$ receiver in the datafusion CSS. The architecture combines a direct-conversion receiver (also known as homodyne or zero-IF receiver) frontend with spectrum sensing functions. The analog RF frontend comprises a wideband antenna, a wideband band-pass filter (BPF), a low-noise amplifier (LNA) and quadrature local oscillators (LOs) and mixers responsible for down-conversion of the sensed passband signal to in-phase and quadrature (I\&Q) baseband signals.

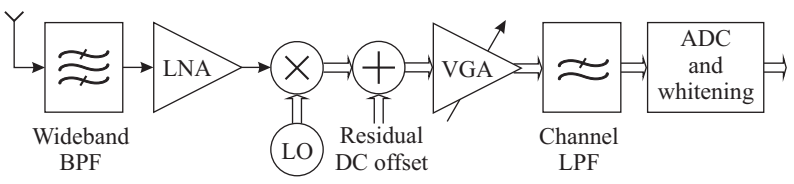

Fig. 1. Receiver architecture considered in the model proposed in [9].

The direct-conversion architecture is the most adequate choice for SDR receivers and monolithic integration, mainly due to its flexibility, low complexity and relaxed image rejection requirements [16], [17]. However, it is prone to impairments such as I\&Q imbalance, flicker noise and DCoffset [17], [18].

The DC-offset is considered the most damaging impairment [18], [19]. It is a direct-current (DC) signal composed of static and dynamic parts, appearing at the mixer output primarily due to LO self-mixing and in-band interfering signals. Without proper compensation, the DC-offset can overload subsequent stages such as amplifiers and analog-to-digital converters (ADCs) [17], [19]. Nonetheless, the adoption of careful circuit design and DC-offset compensation strategies can almost completely eliminate the static DC-offset part, but some residual dynamic part will inevitably remain [17], [20]. The summation block following the mixer in Fig. 1 accounts for this residual dynamic DC-offset.

The baseband I\&Q signals go through a variable gain amplifier (VGA), which is part of an automatic gain control (AGC) mechanism responsible for maintaining the signal within the dynamic range of the ADCs located in the I\&Q signal paths. I\&Q low-pass filters (LPFs) select the desired bandwidth to be sampled and avoids aliasing. Noise whitening subsequently takes place to guarantee that noise samples are kept uncorrelated.

In [9], the receiver depicted in Fig. 1 is modeled taking into account the main operations that influence the spectrum sensing performance, namely: the channel fading and thermal noise, the residual DC-offset, the AGC, filtering, the quantization performed by the ADCs, and the whitening process. A transformed received signal matrix $\mathbf{Y}$ is generated according to these operations, followed by the ordinary computation of the test statistic under analysis.

Aiming at avoiding redundancy, in this section we have omitted the details behind the generation of the transformed matrix Y, since these details are covered in Section IV, where the differences regarding the proposed improved model are highlighted.

\section{IMPROVED RECEIVER MODEL}

The direct-conversion receiver architecture used as reference to construct the proposed spectrum sensing model is shown in Fig. 2. It is quite similar to the architecture depicted in Fig. 1, with the difference that Fig. 2 carries more fidelity with respect to an actual receiver structure [17]. The exchange in position between the LNA and the wideband BPF (WBPF) aims at a reduced noise figure. The digital signal processing (DSP) implements noise whitening, computes the test statistic and performs the decision, also controlling the center frequency of the sensed channel, the DC-offset compensation algorithm, and the VGA that is part of the AGC mechanism. The other blocks in Fig. 2 are self-explanatory.

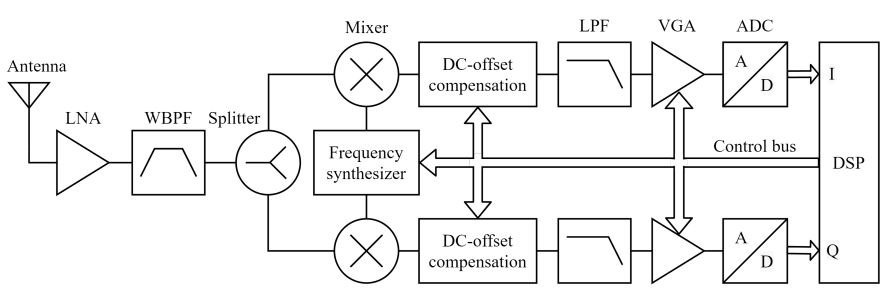

Fig. 2. Homodyne (or zero-IF, or direct-conversion) receiver architecture.

Taking into consideration the same main processes or impairments that degrade the spectrum sensing performance as reported in [9], the FC receiver model can be constructed according to Fig. 3, where $\mathbf{y}_{i}^{\mathrm{T}}$ denotes the $i$-th row, $i=1, \ldots, m$, of the received signal matrix $\mathbf{Y}$ defined in (1).

Matrix $\mathbf{Y}$ is assumed perfectly delivered to the FC, meaning that no bit errors occur during the transmissions of its digitized rows from the SUs to the FC. The influence of such errors is fully investigated in other research; see for instance [21], [22] and references therein.

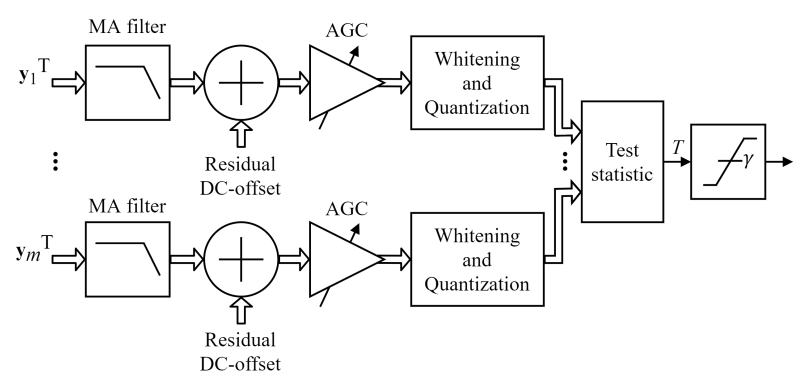

Fig. 3. Fusion center receiver model based on Fig. 2.

To model the effects of filtering on the transmitted and received signals, each row of $\mathbf{Y}$ undergoes moving-average (MA) filtering. Real and imaginary parts of each element in $\mathbf{y}_{i}^{\mathrm{T}}$ are separately filtered. The MA filters have a configurable discrete impulse response length, $L$, which controls the time correlation in the received samples. The MA filter type was chosen for simplicity; any other LPF could be adopted as well.

In each sensing round, the $L$ memory elements of the MA filter are set to zero before filtering, which is equivalent to 
zero-padding the input sequence. Hence, out of $n+L-1$ filtered samples, the first $L-1$ are discarded. In [9], only the samples that form matrices $\mathbf{X}$ and $\mathbf{V}$ are filtered. Here, the samples in $\mathbf{H X}$ and $\mathbf{V}$ are filtered, to take into account the filtering effects on the PU signal at the receiver side as well.

The summation block following the MA filter in Fig. 3 accounts for the residual dynamic DC-offset. In [9] it is added before the MA filter, which affects output samples in a nondesired manner because of the transitory filtering behavior that occurs in the last $L-1$ output samples due to emptying the memory elements at the end of each sensing interval. The residual DC-offset addition after filtering avoids this problem.

Likewise [9], the DC-offset is a complex-valued Gaussian random variable with zero mean, and i.i.d. between sensing rounds. This random variable forms the matrix $\mathbf{D} \in \mathbb{C}^{m \times n}$ that is added to the MA-filtered version of matrix $\mathbf{Y}$. The elements in $\mathbf{D}$ are column-wise equal to one another within a given sensing interval, and row-wise i.i.d. to represent independent DC-offsets among SUs. The variance of the DCoffset samples, $\sigma_{\mathrm{dc}}^{2}$, is determined according to the signal-toDC-offset ratio (SDCR). In $\mathrm{dB}$, it follows that

$$
\mathrm{SDCR}=10 \log _{10}\left(\frac{p_{\mathrm{avg}}}{\sigma_{\mathrm{dc}}^{2}}\right) \text {. }
$$

The combined voltage gain of the LNA and the VGA shown in Fig. 2 is modeled via the AGC block in Fig. 3. At the $i$-th $\mathrm{SU}, i=1,2, \ldots, m$, this gain is given by

$$
g_{i}=\frac{f_{\mathrm{od}} \sqrt{2 n}}{6\left\|\mathbf{y}_{i}\right\|},
$$

where $\|\cdot\|$ denotes the Euclidean norm, and the overdrive factor $f_{\text {od }}$ models different levels of signal clipping caused by actual ADCs. If $f_{\text {od }}$ increases above 1 , clipping occurs with severity proportional to its value. If $f_{\text {od }}<1$, the severity of clipping is progressively reduced as its value becomes far from 1. Clipping acts in a real sample value $y$ according to $y \leftarrow \operatorname{sign}(y) \min (|y|, 0.5)$, where it is implicitly assumed that the dynamic range of the ADCs is $[-0.5,+0.5]$ volts.

The gains defined in (7) have the role of guaranteeing that almost the total signal excursion (six standard deviations) fits the dynamic range of the ADCs, going below or above this range as determined by $f_{\text {od. }}$. In [9] it is also defined a system variable for the ADC dynamic range, but it is not needed because the gains given in (7) attain their role no matter the value of this variable. Further details on the reasoning behind such gain model are given in [9]; they are omitted here for the sake of conciseness.

Whitening is the process of decorrelating the samples contained in a vector such that its covariance matrix becomes the identity matrix [23, p. 130]. Whitening is important in spectrum sensing to decorrelate noise samples, improving the detection performance. In [9], whitening is made after MAfiltering and residual DC-offset addition, before the AGC and the signal clipping plus quantization made by the ADCs. Here, whitening is made after AGC and before quantization (with clipping). This is done in benefit of modeling the use of high resolution ADCs, which is the case of CSS with decision-fusion, in order to avoid performance loss of the
SUs decisions. In CSS with data-fusion, which is the case considered in this paper, whitening has to be made under high resolution, since it is very sensitive to the quantization errors, whereas the samples to be forwarded to the FC can be quantized with lower resolution to reduce the data rate in the control channels. Hence, in the block 'whitening and quantization' shown in Fig. 3 it is subsumed that whitening makes use of high resolution operations (for example, using floating-point arithmetic), while the samples resulted from whitening are quantized with a resolution as small as possible, the smallest sufficient for not causing significant spectrum sensing performance degradation.

The whitening filter [24] matrix $\mathbf{W} \in \mathbb{R}^{n \times n}$ that multiplies the gain-controlled version of $\mathbf{Y}$ is given by

$$
\mathbf{W}=\mathbf{U} \mathbf{L}^{-1},
$$

where $\mathbf{U}$ is an arbitrary orthogonal matrix that can be obtained, for instance, from the singular-value decomposition of the covariance matrix $\mathbf{Q} \in \mathbb{R}^{n \times n}$ associated with the filter impulse response. This matrix has elements

$$
Q_{i j}=a_{|i-j|},
$$

for $i, j=1,2, \ldots, n$, with $\left\{a_{k}\right\}$ denoting the discrete autocorrelation function of the MA filter impulse response, i.e. $a_{k}=1-k / L$ for $k \leq L$, and $a_{k}=0$ otherwise, for $k=0,1, \ldots,(n-1)$. The matrix $\mathbf{L}$ is the lower triangular matrix from the Cholesky decomposition of $\mathbf{Q}$. As highlighted in [9], [24], this whitening process is signal-independent (blind), which renders it easy implementation in practice, since $\mathbf{W}$ can be computed during the system design phase.

In [9], power normalizations were performed in $\mathbf{X}$ and $\mathbf{V}$ after filtering and whitening, as well as in $\mathbf{H}$, to guarantee the desired power levels and channel gains, respectively. We do not apply such normalizations here, since they change the longterm statistics previously assumed for the samples in these matrices. This happens because the normalization factor is the square-root of a time-series average power estimated from the corresponding samples in each sensing round, which is a random variable itself. Here, the desired power levels are guaranteed by just setting the MA filter impulse response as $h_{l}=1 / \sqrt{L}$, for $l=1, \ldots, L$, recalling that the whitening result follows normalized since the whitening filter is derived from the MA filter impulse response.

The present model is based on a receiver (Fig. 2) whose multiple stages in series are necessary and characteristic of a typical direct-conversion structure. They are not peculiar to the model. As can be concluded from the spectrum sensing literature [4], [5], time is shared among the spectrum sensing performed by the SUs, the report of the sensing information to the FC, the decision on the occupation state of the sensed band plus channel assignment, and the access to the channel for data transmission by the secondary network. In practice, the data transmission interval is much larger than the other three intervals, but in any way there exists a trade-off between these intervals so that the target spectrum sensing performance is attained without seriously penalizing the secondary network throughput. Thus, once spectrum sensing is used to drive dynamic spectrum access, its negative contribution to the 
communication latency in the secondary network is expected and it is implicit in the above-mentioned trade-off.

It is important to bear in mind that any time-consuming task (such as whitening and test statistic computation) influences this trade-off, thus influencing latency. The trade-off is also influenced by the time needed to collect the necessary number of samples for spectrum sensing, which in turn depends on the adopted detection technique, on the bandwidth and propagation characteristics of the sensing channel, on the sampling method, and on the number of SUs in cooperation. Delay is also affected by the adopted filtering type (analog or digital) in the signal path. In case of digital filtering, the delay depends on the sampling frequency and filter structure. The delay produced by DSP tasks depends on the processing power, its architecture and clock frequency.

\section{THE ANALYZED DETECTORS}

This section presents the test statistics of the detectors whose performances are analyzed in Section VI, namely: the ED, the LMPIT, the GRCR, the GID and the PRIDe. The computational cost related with the calculation of these test statistics are also presented and discussed.

\section{A. Test statistics}

The ED test statistic [25] in the case of centralized CSS with data-fusion, under the conventional receiver model, is

$$
T_{\mathrm{ED}}=\sum_{i=1}^{m} \frac{1}{\sigma_{i}^{2}} \sum_{j=1}^{n}\left|y_{i j}\right|^{2},
$$

where $y_{i j}$ is the element on the $i$-th row and $j$-th column of the received signal matrix $\mathbf{Y}$ defined in (1), and $\sigma_{i}^{2}$ is the noise variance at the input of the $i$-th SU.

In the case of the implementation-oriented model, the test statistic of the ED becomes

$$
T_{\mathrm{EDio}}=\sum_{i=1}^{m} \frac{1}{g_{i}^{2} \sigma_{i}^{2}} \sum_{j=1}^{n}\left|y_{i j}\right|^{2},
$$

where $g_{i}$, defined in (7), is needed to correct the noise variance information affected by the AGC. This correction was not taken into account in [9], yielding an extremely poor performance of the ED when considering the implementationoriented receiver model.

It is worth highlighting that the ED makes use of $\sigma_{i}^{2}$, which can be embedded into the test statistic, as given in (10) and (11), or into the decision threshold. A common non-realistic practice adopted in the literature, for instance in [9], is to consider that the noise variances across the SUs are the same, that is, $\sigma_{1}^{2}=\sigma_{2}^{2}=\cdots=\sigma_{m}^{2}=\sigma^{2}$. In this case $T_{\mathrm{ED}}$ assumes its mostly known form, $T_{\mathrm{ED}}=\frac{1}{\sigma^{2}} \sum_{i=1}^{m} \sum_{j=1}^{n}\left|y_{i j}\right|^{2}$. When the implementation-oriented model is adopted, it immediately follows that $T_{\text {EDio }}=\frac{1}{\sigma^{2}} \sum_{i=1}^{m} \frac{1}{g_{i}^{2}} \sum_{j=1}^{n}\left|y_{i j}\right|^{2}$.

In the case of the LMPIT detector [10], the test statistic is

$$
T_{\text {LMPIT }}=\sum_{i=1}^{m} \sum_{j=1}^{m}\left|c_{i j}\right|^{2},
$$

where $c_{i j}$ is the element on the $i$-th row and $j$-th column of the matrix $\mathbf{C}=\mathbf{E}^{-1 / 2} \mathbf{R} \mathbf{E}^{-1 / 2}$, for $i, j=1,2, \ldots, m$, where $\mathbf{E}$ is the diagonal matrix in which the diagonal elements are $d_{i i}=r_{i i}$, with $r_{i i}$ denoting the elements on the main diagonal of the sample covariance matrix $\mathbf{R}$ defined in (5).

The test statistic of the GRCR detector [11] is

$$
T_{\mathrm{GRCR}}=\frac{\sum_{i=1}^{m} \sum_{j=1, j \neq i}^{m}\left|r_{i j}\right|}{\sum_{i=1}^{m} r_{i i}},
$$

where $r_{i j}$ is the element on the $i$-th row and $j$-th column of the matrix $\mathbf{R}$.

Finally, the test statistics of the GID [12] and the PRIDe [13] detectors are respectively computed according to

$$
\begin{gathered}
T_{\mathrm{GID}}=\frac{\sum_{i=1}^{m^{2}}\left|r_{i}\right|}{\sum_{i=1}^{m^{2}} \sum_{j=1}^{m^{2}}\left|r_{i}-r_{j}\right|}, \\
T_{\mathrm{PRIDe}}=\frac{\sum_{i=1}^{m^{2}}\left|r_{i}\right|}{\sum_{i=1}^{m^{2}}\left|r_{i}-\bar{r}\right|},
\end{gathered}
$$

where $r_{i}$, for $i=1,2, \ldots, m^{2}$, is the $i$-th element of the vector $\mathbf{r}$ formed by stacking all columns of $\mathbf{R}$, and $\bar{r}=\left(1 / m^{2}\right) \sum_{i=1}^{m^{2}} r_{i}$.

As presented in the next subsection, the detectors LMPIT, GRCR, GID and PRIDe are the blind detectors of less computational complexity known to date, to the best of the authors knowledge.

\section{B. Computational complexities}

The ED test statistic (10) is the less complex detector known so far. Its computational cost is dominated by the $\mathrm{nm}$ multiplications, thus yielding a complexity of $O(\mathrm{~nm})$. However, one must be aware that the final computational burden related to the ED will also depend on the method used to estimate the noise variance, which is a known parameter in (10), but known within the estimation error range in practice.

The complexities of the test statistics LMPIT (12), GRCR (13), GID (14), and PRIDe (15) are dominated by the complexity associated with the computation of the sample covariance matrix $\mathbf{R}$, which is $O\left(\mathrm{~nm}^{2}\right)$ [26], [27]. In the case of the LMPIT, it deserves emphasis the fact that the matrix $\mathbf{C}$ is formed by a matrix multiplication, being the operation $\mathbf{E}^{-1 / 2}$ quite simple, given that $\mathbf{E}$ is diagonal. Hence, most of the computational complexity associated with the LMPIT indeed resides in the computation of $\mathbf{R}$.

Since the number $m$ of SUs in cooperation is small in practice, it can be concluded that the computational cost to calculate the test statistics of the detectors LMPIT, GRCR, GID and PRIDe is slightly higher than in the case of the ED, when the ED uses prior information on the noise variance. If the ED estimates the noise variance on-the-fly, the computational cost 
of doing such estimation must be added, eventually making the ED more complex than the other detectors in analysis.

The noise whitening, which is not considered in the conventional model, is a necessary operation in the proposed implementation-oriented model, since it is responsible for decorrelating the noise samples present in the signal due to filtering effects in the signal path. The computational complexity related to the whitening approach adopted in this work costs $O\left(n^{2} m\right)$, which is from the matrix multiplication between $\mathbf{W} \in \mathbb{R}^{n \times n}$ and $\mathbf{Y}^{\mathrm{T}} \in \mathbb{C}^{n \times m}$.

The blocks preceding whitening are common in SDR receivers, namely: filtering, down-conversion and analog-todigital conversion. Their implementation complexities are not related to the computational burden of the proposed model. Instead, they are related with the actual implementation complexity of the receiver architecture depicted in Fig. 2.

The computations of the test statistic and the noise whitening contribute with the delay of the spectrum sensing process, as discussed in the last two paragraphs of Section IV.

\section{NUMERICAL RESULTS}

The numerical results presented in this section show graphs of $P_{\mathrm{d}}$ versus the most relevant system parameters, assuming a constant $P_{\text {fa }}=0.1$ [8]. Each point on all curves has been determined from 50000 Monte Carlo computer simulation runs, which corresponds to the generation of the same number of each test statistic under $\mathcal{H}_{0}$ and $\mathcal{H}_{1}$. The MATLAB simulation code used to generate the results is available at [28].

The $95 \%$ confidence intervals (CIs) achieved using the above number of runs are shown in Fig. 4, which clearly unveils accurate estimates of $P_{\mathrm{d}}$. These CIs were computed using the binomial proportion confidence interval for single proportion, a method that is based on the number of successes in independent Bernoulli trials. Since the noise and fading channel gains are independent between sensing events, and the SUs decisions are independent from each other, the spectrum sensing results are Bernoulli random variables and the number of detection events during the Monte Carlo runs has a Binomial distribution. Hence, the above method is adequate for CI calculation. The Matlab function 'binofit' has been used to compute the CIs. It adopts the Clopper-Pearson method, also called 'exact binomial' method [29].

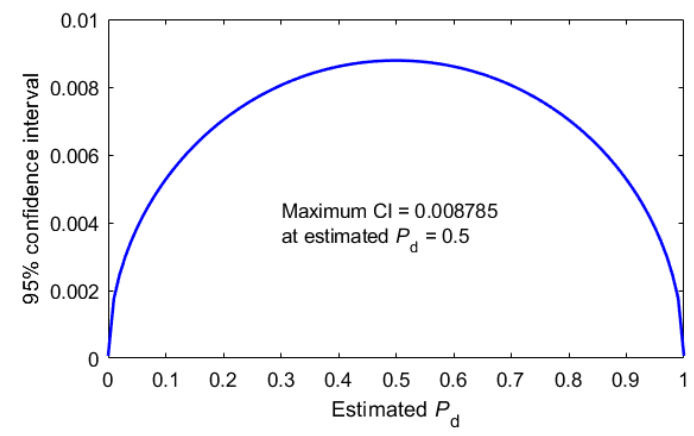

Fig. 4. Confidence interval of the Monte Carlo simulations.

Unless otherwise stated, the system parameters, when fixed, are $s=1, m=6, n=140, \mathrm{SNR}=-10 \mathrm{~dB}, \mathrm{SDCR}=5 \mathrm{~dB}$,
$N_{\mathrm{q}}=8(3$ bits $), f_{\mathrm{od}}=1.2, L=n / 10, \rho_{\mathrm{S}}=0.9, \rho_{\mathrm{N}}=0.2$, $\mu_{K}=1.88 \mathrm{~dB}, \sigma_{K}=4.13 \mathrm{~dB}$, and $P_{\mathrm{fa}}=0.1$. The SNR or $n$ has been set to yield the standardized [8] value of $P_{\mathrm{d}} \approx 0.9$ around the mid-range of the parameter under test, for the best detector under the conventional model. Using this procedure, the influence of higher and lower values of the parameter under test can be easily seen in the graphs.

The quantities of PUs and SUs ( $s=1, m=6)$ were chosen to match situations that are likely to occur in practice, namely a single PU transmitter per coverage area and a small number os SUs in cooperation to save control channel resources. Moreover, the cooperation diversity grows in a diminishing-return fashion, meaning that the spectrum sensing performance does not improve linearly on $m$. The amount of 140 samples was chosen for being enough to yield the typical standardized target performances $\left(P_{\mathrm{fa}}=0.1\right.$ and $\left.P_{\mathrm{d}}=0.9\right)$ at a significantly small SNR regime of $-10 \mathrm{~dB}$ in most of the situations analyzed. The conservative $\mathrm{SDCR}=5 \mathrm{~dB}$ has been chosen because the residual SDCR can be as high as 15 to $30 \mathrm{~dB}$ when the DC-offset compensation circuit is properly designed [30]. The number of 8 quantization levels is almost as good as infinite quantization, which can be observed in the results hereafter. An overdrive factor of 1.2 and an MA filter impulse response length of $n / 10$ are adequate choices for yielding close-to-the-best performances for all detectors, as also observed in the results ahead. The fractions of noise and received signal level variations were arbitrarily chosen, with the fraction of noise variation smaller than the one related to the signal, aiming at mimicking a more likely situation in practice (received signal powers vary more due to variable distances between transmitters and spectrum sensors; thermal noise fluctuations are considerably smaller due to smaller variations in the ambient temperature and small circuit uncalibration or interfering signals). The Rice factor parameters were chosen to represent the spectrum sensing scenario of more practical appeal, which corresponds to a urban environment.

Fig. 5 shows $P_{\mathrm{d}}$ versus the average SNR across the SUs. As expected, all detectors attain performance improvement as the SNR increases. The ED has a slightly superior performance, followed by the PRIDe. The LMPIT and the GRCR attain comparable performances, which are superior to the GID at higher SNR regimes, whereas at lower SNRs the GID wins. The performances follow approximately equal patterns from the conventional to the implementation-oriented model, but an overall decrease can be observed in the latter due to the impairments not present in the former.

The results shown in Fig. 5 are representative of an outcome also observed in the other figures shown hereafter, which is the different behaviors among the detectors when a system parameter (the SNR in the case of Fig. 5) is varied. It may happen that no explicit cause for such different behaviors can be highlighted. In other words, it is expected that different detectors perform unequally under a given system setup, and it is also expected that the variation of a given parameter will produce different degrees of performance variation in different detectors, since their test statistics operate the received signal differently from each other. 

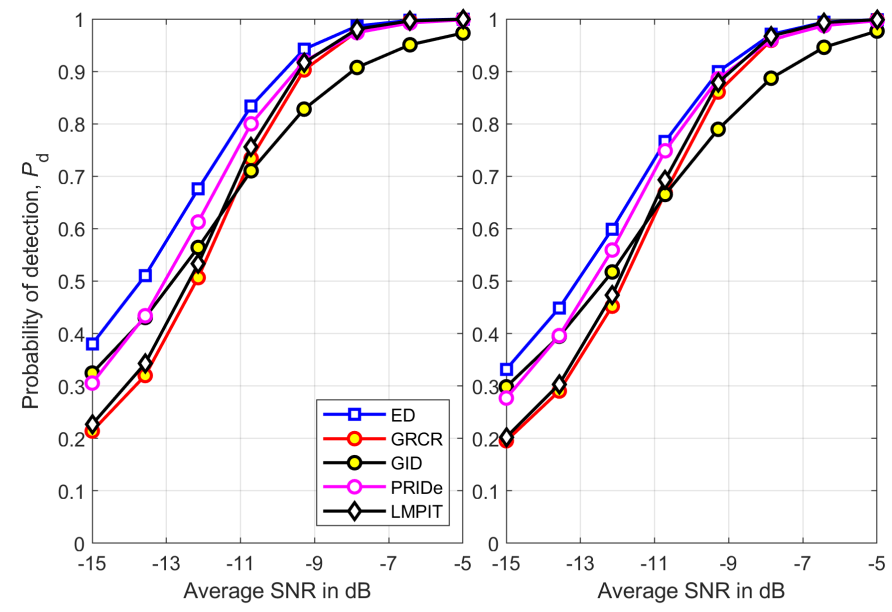

Fig. 5. $P_{\mathrm{d}}$ versus average SNR: conventional model (left), implementationoriented model (right).

Fig. 6 shows $P_{\mathrm{d}}$ versus the number of samples $n$ collected by each SU. All detectors attain performance improvement as $n$ increases, which is an expected outcome since a larger $n$ can be translated into a larger sensing interval if the sampling rate is unaltered. All but the ED yield performances following approximately equal patterns from the conventional to the implementation-oriented model, with an overall decrease observed in the latter. For larger values of $n$, the ED unveiled a more prominent performance penalty from the conventional to the implementation-oriented model. The LMPIT and the GRCR attain performances close to each other and superior to the GID at higher values of $n$, whereas at the GID wins for smaller values of $n$.

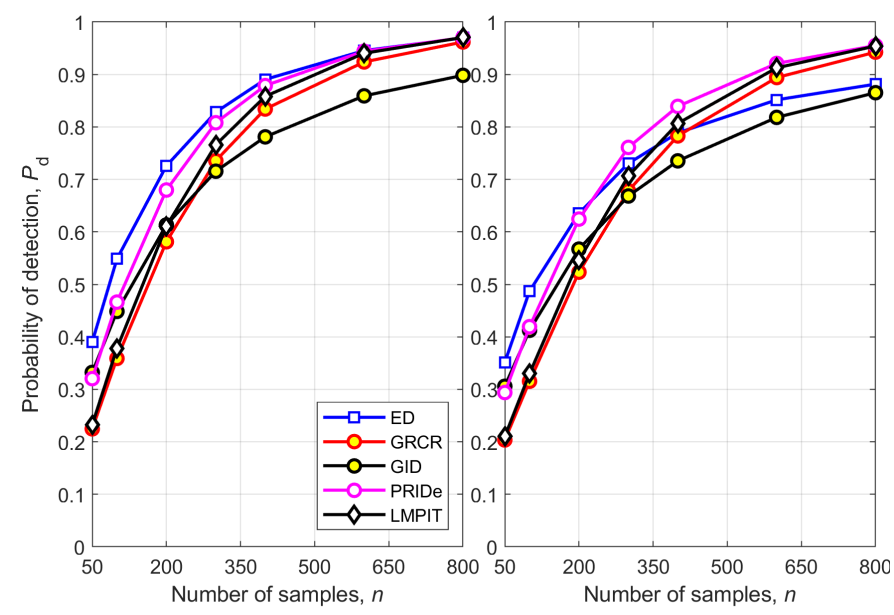

Fig. 6. $P_{\mathrm{d}}$ versus the number of samples, $n$ : conventional model (left), implementation-oriented model (right). SNR $=-12.5 \mathrm{~dB}$.

The variation in $P_{\mathrm{d}}$ according to the number of SUs in cooperation, $m$, is demonstrated in Fig. 7. As happens with the SNR and $n$, the performances of all detectors improve when $m$ increases, but not linearly. This improvement is mainly a consequence of an increased spatial diversity gain achieved when more SUs cooperate. The improvement of the ED is less pronounced than the others. All performances follow approximately equal patterns from the conventional to the implementation-oriented model, with an overall penalty observed in the latter. The penalty for the ED is larger.

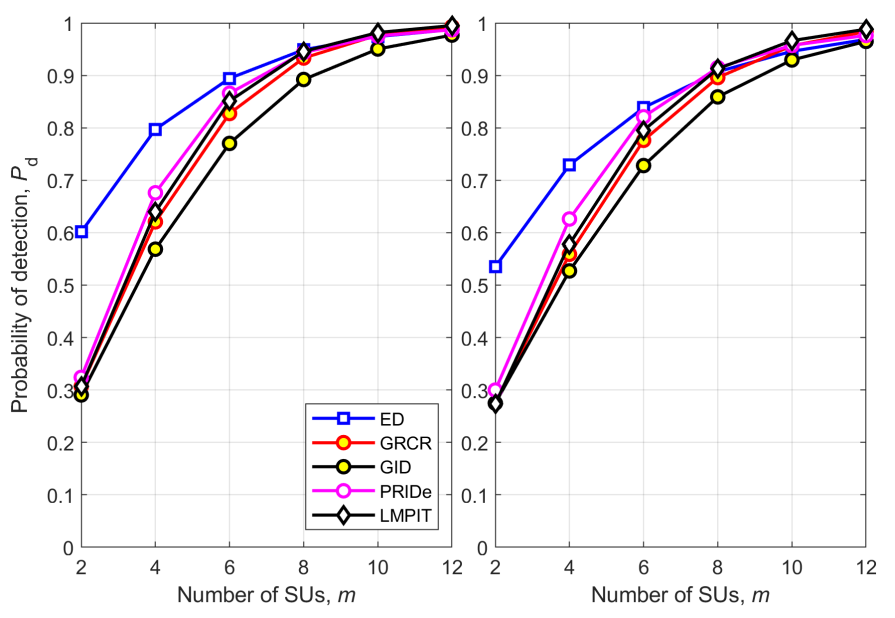

Fig. 7. $P_{\mathrm{d}}$ versus the number of SUs, $m$ : conventional model (left), implementation-oriented model (right).

The influence of the number of PUs, $s$, on $P_{\mathrm{d}}$ is shown in Fig. 8. The detectors in analysis unveil quite similar patterns from the conventional to the implementation-oriented model, once again showing an overall performance penalty in the latter case. Though the ED is quite insensitive to the number of PUs, its performance penalty is more pronounced from the former to the latter model. The small sensitivity of the ED to the number os PUs relates to the fact that the detection performance is based on the overall energy of the received signals, which does not change with $s$ for a fixed SNR. Due to unknown reasons, the small sensitivity to $s$ is also a characteristic of the GID. The PRIDe is slightly more sensitive, whereas the LMPIT and the GRCR have their performances considerably more affected by variations in $s$. Nonetheless, the unequal behaviors of the detectors is an expected outcome due to the different ways in which the received signal is processed by each test statistic, as already explained.

Fig. 9 presents how the impulse response length $L$ of the MA filters affects $P_{\mathrm{d}}$. In the graph on the left it can be noticed that the performance does not change with $L$ (small fluctuations can be observed due to the normal randomness of the Monte Carlo simulation), since this graph refers to the conventional receiver model, which does not consider any filtering effect. When the implementation-oriented model is adopted, an overall performance loss is observed, which is more pronounced in the case of the ED for larger values of $L$. The other detectors are affected in a similar way, but in all detectors it is observed that larger values of $L$ tend to produce higher performance losses. This is a consequence of the higher time correlation (lower bandwidth) of the filtered signal, given that uncorrelatedness is not completely restored through whitening. When $L=1$ there is no filtering and, as a consequence, there is no whitening. However, in this situation the performance loss can be higher than for other values of 


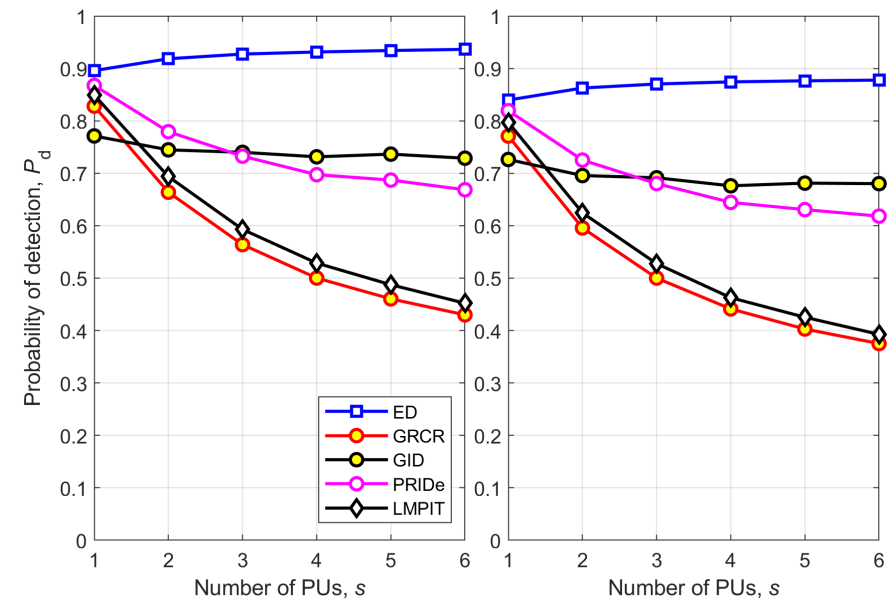

Fig. 8. $\quad P_{\mathrm{d}}$ versus the number of PUs, $s$ : conventional model (left), implementation-oriented model (right). $n=120$.

$L$ due to the influence of the DC-offset that, besides possible overload of subsequent stages, also increases time correlation. In other words, if the SDCR were smaller, the performance would have been more penalized for $L=1$, improving for values of $L$ a little larger, then worsening again for even larger $L$. Results such as this have been observed, but were omitted for conciseness.

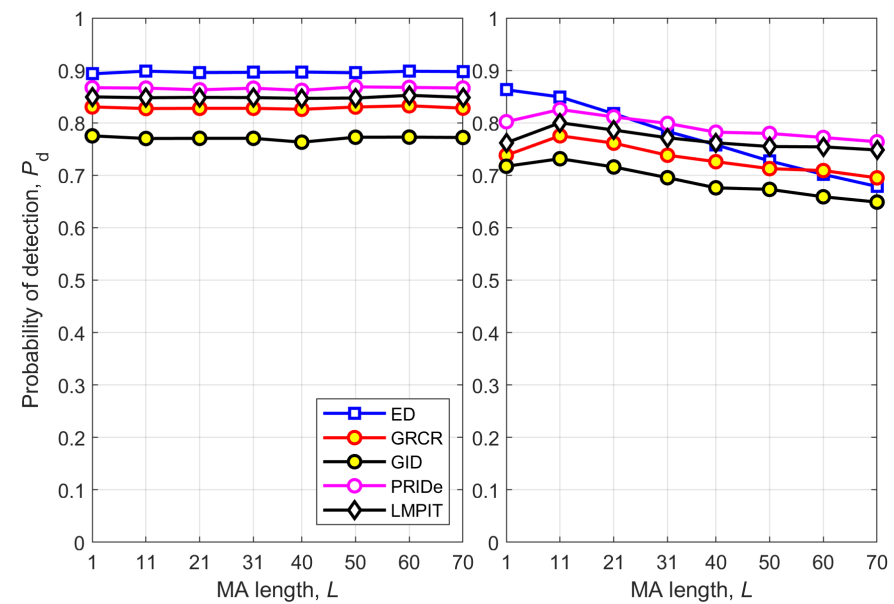

Fig. 9. $P_{\mathrm{d}}$ versus the length of the MA filter impulse response, $L$ : conventional model (left), implementation-oriented model (right).

The effects on $P_{\mathrm{d}}$ caused by different values of SDCR are shown in Fig. 10. The graph on the left is equal to the one shown in Fig. 9, and the same comments apply. In the rightside graph it can be observed an expected behavior, which is a performance loss for all detectors at lower SDCRs. Above an SDCR of $-5 \mathrm{~dB}$, the loss variation becomes negligible. Once again, an overall performance penalty is observed from the conventional to the implementation-oriented model, since the former does not take the DC-offset into account.

Fig. 11 illustrates the effect of the ADC overdrive factor, $f_{\text {od }}$, on $P_{\mathrm{d}}$. The graph on the left is equal to the ones shown in Figs. 9 and 10, the same comments applying again. It

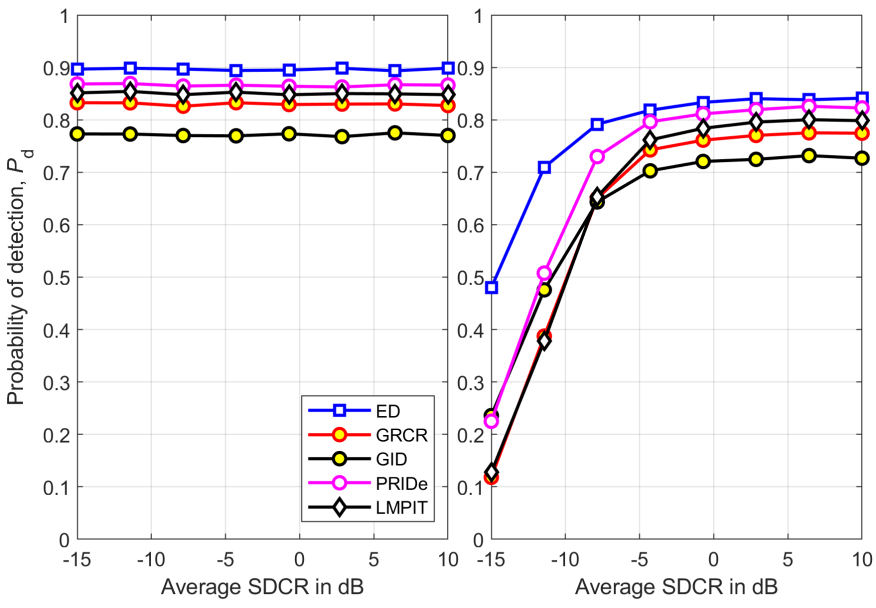

Fig. 10. $P_{\mathrm{d}}$ versus average SDCR: conventional model (left), implementationoriented model (right).

can be noticed that the performance variations with $f_{\text {od }}$ are quite small, except in the case of the ED. For all detectors there are concave curve shapes. Smaller values of $f_{\text {od }}$ make the signal excursion smaller than the dynamic range of the ADC, meaning that such signal crosses a smaller number of quantization levels, which reduces performance. High values of $f_{\text {od }}$ make the signal excursion larger than the dynamic range of the ADC, causing clipping and, thus, also reducing performance. The larger influence of the $f_{\text {od }}$ on the ED is due to the fact that quantization also affects the noise variance information that is used in the ED test statistic, which represents another source of performance loss in addition to insufficient quantization (when $f_{\text {od }}$ is small) or severe clipping (when $f_{\text {od }}$ is large).

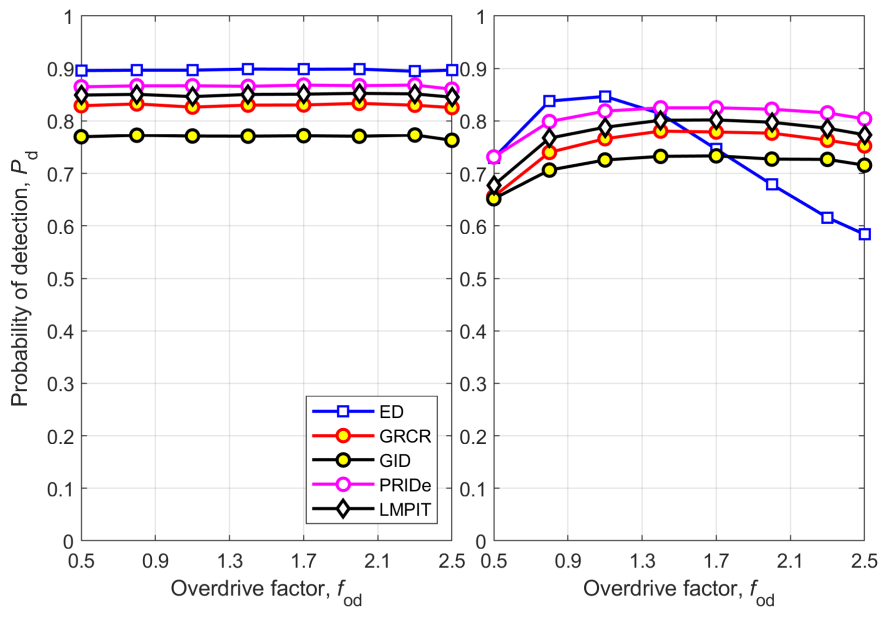

Fig. 11. $P_{\mathrm{d}}$ versus the overdrive factor, $f_{\text {od }}$ : conventional model (left), implementation-oriented model (right).

Lastly, the influence of the number of quantization levels, $N_{\mathrm{q}}$, on $P_{\mathrm{d}}$ is shown in Fig. 12. The number of quantization levels is expressed in terms of the corresponding number of bits, which is $\log _{2} N_{\mathrm{q}}$. Once again, the graph on the left is equal to the ones shown in Figs. 9, 10 and 11, the same 
comments applying. A performance loss is again observed from the conventional to the implementation-oriented model, since the former implicitly uses infinity quantization. As awaited, the spectrum sensing performances of all detectors improve if $N_{\mathrm{q}}$ increases, as shown in the right-side graph. The improvement regarding the ED is more pronounced than in the case of the other detectors, with the ED yielding the poorest performance for 1-bit resolution, outperforming the other detectors when a 3-bit resolution or more is used. It can be seen that the performance improvement is marginal beyond 3 bits (8 quantization levels), a result also verified in [9]. Recall that we are considering the quantization of the samples transmitted from the SUs to the FC, not the one associated with other digital signal processing operations made by each SU, such as digital filtering and whitening. Also recall that a small number of bits representing the samples forwarded to the FC is beneficial to the reduction of the control channel bandwidth usage.

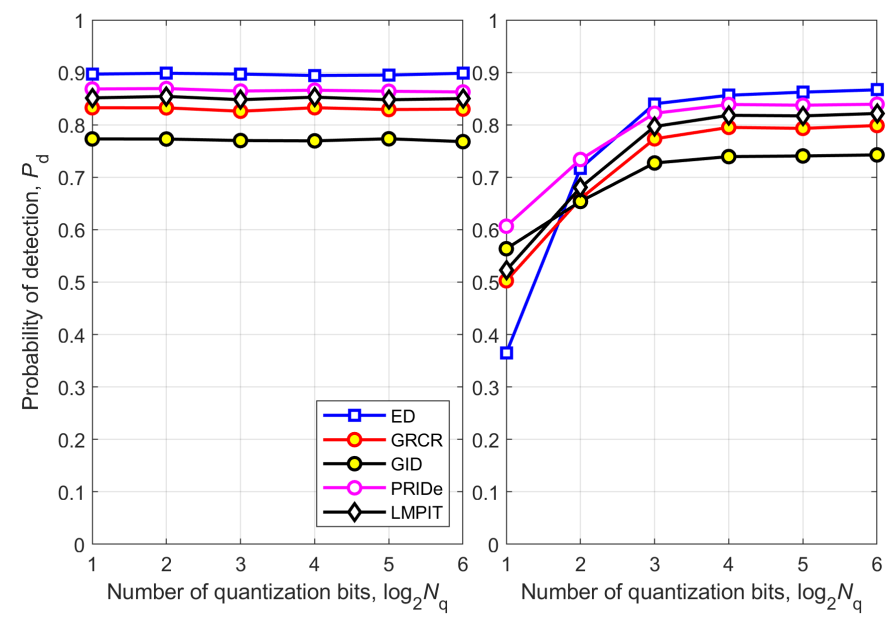

Fig. 12. $P_{\mathrm{d}}$ versus the number of quantization bits, $\log _{2} N_{\mathrm{q}}$ : conventional model (left), implementation-oriented model (right).

\section{CONCLUSIONS}

The research documented in this paper has improved the implementation-oriented model proposed in [9], applying it to assess the performances of state-of-the-art detectors whose computations of the test statistics are among the less complex known to date, which are the GRCR, the GID, the PRIDe and the LMPIT. The ED has been also included as a benchmark. The sensing channel model has been also improved with respect to the one adopted in [9], taking into account a morphology-dependent random Rice factor, as well as timevariant noise and received signal levels.

It has been shown that the performances of all detectors are overestimated if the conventional model is adopted. The ED is the one whose performance is more penalized by the operations made in the implementation-oriented model. Moreover, under this model, the ED needs to apply the exact AGC gain in the composition of its test statistic, besides the exact noise variance information. If any of these data are inaccurate, the ED may become useless.
Regarding the performances of the detectors GRCR, GID, PRIDe and LMPIT, it has been observed that all of them follow approximately the same pattern in terms of the influence of the implementation-oriented model operations, with an advantage (or at least approximate equality) in performance of the PRIDe in the majority of the situations analyzed, for example as shown in Fig. 5 above $\mathrm{SNR} \approx-14 \mathrm{~dB}$, in Fig. 6 above $n \approx 70$, in Fig. 7 for all $m$, in Fig. 8 below $s=3$, and in Figs. 9-12 for all varied parameter values. The GRCR and the LMPIT have performances close to each other, beating the GID in some circumstances, but being beaten by the GID in others. For example, in Fig. 5 the GRCR and the LMPIT outperform the GID for SNR values above $\approx-12 \mathrm{~dB}$, whereas the GID wins below this SNR. Similar behaviors can be identified in Fig. 6 for the crossing point around $n=200$, in Fig. 8 for the crossing point between $s=1$ and $s=2$, in Fig. 10 (right) for the crossing point around SDCR $=-8 \mathrm{~dB}$, and in Fig. 12 (right) for the crossing point around 2 bits.

Besides the system parameters that obviously influence the spectrum sensing performance when they are varied, namely the SNR, the number of SUs, the number of PUs (in less extent) and the number of samples, it has been verified that the SDCR and $N_{\mathrm{q}}$ also produce large performance variations. However, the use of common DC-offset compensation strategies can increase the SDCR to values in which the performance degradation caused by this impairment becomes negligible. In regard to $N_{\mathrm{q}}$, it has been shown that 3 bits are enough to represent the samples transmitted from the SUs to the FC, which represents considerable control channel bandwidth savings.

The sensed signal bandwidth, indirectly modeled by the impulse response length $L$ of the filters used in the implementation-oriented model, has a small influence on the spectrum sensing performance, since this influence is almost removed by means of whitening. The ED, however, is more sensitive to the variation of this length. The higher sensitivity to the variation of the overdrive factor $f_{\text {od }}$ is also a characteristic of the ED.

It has been also observed different behaviors among the detectors when a system parameter is varied. Sometimes, no explicit cause for such different behaviors can be highlighted. Put in another way, it is expected that different detectors perform unequally under a given system setup, and it is also expected that the variation of a given parameter will produce different degrees of performance variation in different detectors, since their test statistics operate the received signal differently from each other.

Some of the conclusions reported here were also observed in [9]; others have not. But even the conclusions that match are supported by different numerical values, since the implementation-oriented model considered in this paper is an improved and modified version of the one described in [9]. It must be taken into account that the detectors considered in [9] are different from those assessed here, with the exception of the ED, which has been considered in both research, but under different test statistics.

A natural continuity of this research is the implementation of the detectors addressed in this paper using a real hardware, 
for example the universal software radio peripheral (USRP) device, aiming at the verification and validation of the present implementation-oriented model.

\section{REFERENCES}

[1] Federal Communications Commission, FCC, Spectrum Policy Task Force Report. Federal Communications Commission, 2002. [Online]. Available: https://books.google.com.br/books?id=p9MQtwAACAAJ.

[2] M. A. McHenry, P. A. Tenhula, D. McCloskey, D. A. Roberson, and C. S. Hood, "Chicago spectrum occupancy measurements \& analysis and a long-term studies proposal," in Proc. of the first Int. workshop on technology and policy for accessing spectrum. ACM, 2006, doi: $10.1145 / 1234388.1234389$.

[3] J. Mitola III and G. Q. Maguire Jr., "Cognitive radio: making software radios more personal," IEEE Pers. Commun., vol. 6, no. 4, pp. 13-18, Aug 1999, doi: 10.1109/98.788210.

[4] I. F. Akyildiz, B. F. Lo, and R. Balakrishnan, "Cooperative spectrum sensing in cognitive radio networks: A survey," $E l$ sevier Physical Comm., vol. 4, pp. 40-62, mar 2011, doi: 10.1016/j.phycom.2010.12.003.

[5] Y. Arjoune and N. Kaabouch, "A comprehensive survey on spectrum sensing in cognitive radio networks: Recent advances, new challenges, and future research directions," Sensors, vol. 19, no. 1, 2019 , doi: 10.3390/s19010126. [Online]. Available: http://www.mdpi.com/ $1424-8220 / 19 / 1 / 126$.

[6] A. Nasser, H. Al Haj Hassan, J. Abou Chaaya, A. Mansour, and K.-C. Yao, "Spectrum sensing for cognitive radio: Recent advances and future challenge," Sensors, vol. 21, no. 7, 2021, doi: 10.3390/s21072408. [Online]. Available: https://www.mdpi.com/1424-8220/21/7/2408.

[7] W. Dias, A. Ferreira, R. Kagami, J. S. Ferreira, D. Silva, and L. L. Mendes, "5G-Range: A transceiver for remote areas based on software-defined radio," in 2020 European Conference on Networks and Communications (EuCNC), 2020, pp. 100-104, doi: 10.1109/EuCNC48522.2020.9200925.

[8] The Institute of Electrical and Electronic Engineers, IEEE. (2011) IEEE 802 Part 22: Cognitive Wireless RAN Medium Access Control (MAC) and Physical Layer (PHY) Specifications: Policies and Procedures for Operation in the TV Bands. [Online]. Available: http://standards.ieee.org/getieee802/download/802.22-2011.pdf.

[9] D. A. Guimarães and R. A. A. de Souza, "Implementation-oriented model for centralized data-fusion cooperative spectrum sensing," IEEE Commun. Lett., vol. 16, no. 11, pp. 1804-1807, 2012, doi: 10.1109/LCOMM.2012.092112.121614.

[10] D. Ramirez, J. Via, I. Santamaria, and L. L. Scharf, "Locally most powerful invariant tests for correlation and sphericity of gaussian vectors," IEEE Transactions on Information Theory, vol. 59, no. 4, pp. 21282141, April 2013, doi: 10.1109/TIT.2012.2232705.

[11] D. A. Guimarães, "Robust test statistic for cooperative spectrum sensing based on the Gerschgorin circle theorem," IEEE Access, vol. 6, pp. 2445-2456, 2018, doi: 10.1109/ACCESS.2017.2783443.

[12] —, "Gini index inspired robust detector for spectrum sensing over Ricean channels," Electron. Lett., November 2018, doi: 10.1049/el.2018.7375.

[13] — "Pietra-Ricci index detector for centralized data fusion cooperative spectrum sensing," IEEE Trans. Veh. Technol., vol. 69, no. 10, pp. 12354-12358, 2020, doi: 10.1109/TVT.2020.3009440.

[14] B. Nadler, F. Penna, and R. Garello, "Performance of eigenvalue-based signal detectors with known and unknown noise level," in IEEE Int. Conf. Commun., Jun 2011, pp. 1-5, doi: 10.1109/icc.2011.5963473.

[15] S. Zhu, T. S. Ghazaany, S. M. R. Jones, R. A. Abd-Alhameed, J. M. Noras, T. Van Buren, J. Wilson, T. Suggett, and S. Marker, "Probability distribution of Rician $K$-factor in urban, suburban and rural areas using real-world captured data," IEEE Trans. Antennas Propag., vol. 62, no. 7 , pp. 3835-3839, Jul 2014, doi: 10.1109/TAP.2014.2318072.

[16] J. Laskar and S. Chakraborty, Modern receiver front-ends: Systems, Circuits, and Integration. Hoboken, NJ, USA: Wiley-Interscience, 2004. [Online]. Available: http://www.loc.gov/catdir/bios/wiley046/ 2003019188.html.

[17] S. Spiridon, Toward 5G Software Defined Radio Receiver Front-Ends, ser. SpringerBriefs in Electrical and Computer Engineering. Springer International Publishing, 2016, doi: 10.1007/978-3-319-32759-4.

[18] B. Razavi, "Design considerations for direct-conversion receivers," IEEE Transactions on Circuits and Systems-Part II: Analog and Digital Signal Processing, vol. 44, no. 6, pp. 428-435, Jun. 1997, doi: $10.1109 / 82.592569$
[19] R. Svitek, and S. Raman, "DC offsets in direct-conversion receivers: characterization and implications," IEEE Microw. Mag., vol. 6, no. 3, pp. 76-86, Sep. 2005, doi: 10.1109/MMW.2005.1511916.

[20] M. Keshavarzi, A. Mohammadi, and A. Abdipour, "Characterization and compensation of DC offset on adaptive MIMO direct conversion transceivers," IEICE Trans. Commun., vol. E94-B, no. 1, pp. 253-261, Jan. 2011, doi: 10.1109/WIRELESSVITAE.2009.5172451.

[21] L. S. Costa, D. A. Guimarães, and R. A. A. de Souza, "Performance of the Gerschgorin radii and centers ratio detector for cooperative spectrum sensing under burst control channel errors," J. Commun. and Inf. Systems, vol. 34, no. 1, pp. 141-153, May 2019, doi: 10.14209/jcis.2019.15. [Online]. Available: https://jcis.sbrt.org.br/jcis/ article/view/661.

[22] L. S. Costa, D. A. Guimarães, R. A. A. De Souza, and R. C. D. V. Bomfin, "Cooperative spectrum sensing with coded and uncoded decision fusion under correlated shadowed fading report channels," Sensors, vol. 19, no. 1, 2019, doi: 10.3390/s19010051. [Online]. Available: https://www.mdpi.com/1424-8220/19/1/51.

[23] A. Cichocki, Adaptive Blind Signal and Image Processing: Learning Algorithms and Applications. John Wiley \& Sons Incorporated, 2002. [Online]. Available: https://www.wiley.com/en-us/Adaptive+ Blind+Signal+and+Image+Processing $\% 3 \mathrm{~A}+$ Learning+Algorithms+ and+Applications-p-9780470845899

[24] R. Wang and M. Tao, "Blind spectrum sensing by information theoretic criteria for cognitive radios," IEEE Trans. Veh. Technol., vol. 59, no. 8, pp. 3806-3817, 2010, doi: 10.1109/TVT.2010.2065250.

[25] H. Urkowitz, "Energy detection of unknown deterministic signals," Proceedings of the IEEE, vol. 55, no. 4, pp. 523-531, 1967, doi: 10.1109/PROC.1967.5573.

[26] L. Huang, T. Long, and S. Wu, "Source enumeration for high-resolution array processing using improved Gerschgorin radii without eigendecomposition," IEEE Transactions on Signal Processing, vol. 56, no. 12, pp. 5916-5925, 2008, doi: 10.1109/TSP.2008.929331.

[27] X. Chen, M. R. Lyu, and I. King, "Toward efficient and accurate covariance matrix estimation on compressed data," in International Conference on Machine Learning. PMLR, 2017, pp. 767-776. [Online]. Available: http://proceedings.mlr.press/v70/chen17g.html

[28] D. A. Guimarães and E. J. T. Pereira, "Performance of detectors for spectrum sensing using a direct-conversion receiver model," May 2021, doi: 10.24433/CO.0788735.v1. [Online]. Available: https: //codeocean.com/capsule/1153872/tree/v1.

[29] R. Newcombe, "Two-sided confidence intervals for the single proportion: comparison of seven methods." Statistics in medicine, vol. 17 8, pp. 857-72, 1998. [Online]. Available: https://onlinelibrary.wiley.com/doi/10.1002/(SICI) 1097-0258(19980430)17:8\%3C857::AID-SIM777\%3E3.0.CO;2-E

[30] M. Krueger, R. Denk, and B. Yang, "Good and bad training sequences for zero IF sampling EDGE receivers," in IEEE Int. Conf. on Acoust., Speech, and Signal Process., vol. 4, May 2004, pp. iv-1033, doi: 10.1109/ICASSP.2004.1327006.

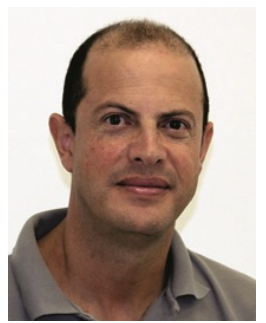

Dayan Adionel Guimarães received an MSc and a $\mathrm{PhD}$ in Electrical Engineering from the State University of Campinas (Unicamp), Brazil, in 1998 and 2003, respectively. He is a Researcher and Senior Lecturer in the National Institute of Telecommunications (Inatel), Brazil. His research focuses the general aspects of wireless communications, specifically radio propagation, digital transmission, dynamic spectrum access, and convex optimization and signal processing applied to telecommunications.

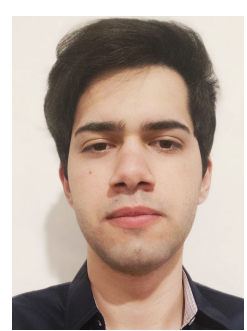

Elivander Judas Tadeu Pereira received the degree of Bachelor in Engineering (2018) and the MSc in Telecommunications (2020) from the National Institute of Telecommunications (Inatel), Brazil. $\mathrm{He}$ is currently working towards his Doctorate on Telecommunications at Inatel. His research interests are mobile communications, digital transmission, cognitive radio, statistics and signal processing. 